\title{
Realization of Self-Rotating Droplets Based on Liquid Metal
}

\author{
Zhanlong Wang, Xiaohe Wang, Qing Miao, and Ya-Pu Zhao*
}

\begin{abstract}
Owing to their fascinating characteristics, liquid metals (LMs) have attracted increasing attention from the scientific community, and are a potential material for various applications. A novel phenomenon is reported in which an acid droplet spontaneously rotates on the surface of an LM. The experimental results show that this phenomenon originates from the collective motion of bubbles generated by the chemical reactions between the droplet and the LM. The angular velocity of the droplet rotation is on the order of $10^{7} \mathrm{rad} \mathrm{s}^{-1}$, which is much higher than that driven by other mechanisms. Under different conditions, the period of the droplet differs, and it increases with the $\mathrm{pH}$ and radius of the acid droplet. The theoretical results indicate the dominant factors and the characterized angular velocity, which agree well with the experimental data. This phenomenon demonstrate that the general particles can also induce special spatial-temporal patterns, and opens up a new field for the application of LMs.
\end{abstract}

drops, Hill et al. ${ }^{[18,19]}$ observed an unusual mode that appears to be a hybrid of binary fission and Taylor cone-jet fission. Bouillant et al. ${ }^{[20]}$ designed a Leidenfrost wheel that can automatically run on a homogeneous hot solid surface by employing the spatial inhomogeneity of heat flow. Koyano et al. ${ }^{[25]}$ fabricated a rotor that is made up of two camphor disks, and the maximum angular velocity reached $20 \mathrm{rad} \mathrm{s}^{-1}$. The driving force originates from the surface tension gradient induced by the dissolution of camphor. Oswald et al. ${ }^{[21,22]}$ reproduced the Lehmann effect, ${ }^{[26]}$ where the internal texture of drops of a cholesteric liquid crystal between two glass plates can be put into motion, such as spinning, when heated from below. These works deepened the understanding of rotational droplets and broadened its applications.

Liquid metal (LM) has attracted increasing attention owing to its significant application prospects, ${ }^{[1-3]}$ for example, the fabrication of flexible electronics, ${ }^{[4]}$ realizing the spontaneous movement of droplets. ${ }^{[5,6]}$ Achieving automatic motion of droplets, including translation and rotation, is the goal of many researchers in the areas of wetting, ${ }^{[7,8]}$ microfluidic chips, ${ }^{[9]}$ self-cleaning surfaces, ${ }^{[10]}$ etc. Potential applications of this technology include liquid motors, ${ }^{[11]}$ wheeled robots, ${ }^{[12]}$ and microfluidic components. ${ }^{[1,2]}$ The study of droplet rotation can be traced back to the experiments conducted by Plateau in 1863. ${ }^{[13]}$ He observed that a droplet experiences a process of two-lobed, three-lobed, and even four-lobed configurations as the angular velocity increases. ${ }^{[13]}$ Since then, the equilibrium shapes of spinning droplets have been successfully employed to describe the structure and deformation of various systems ranging from atomic nuclei to astronomical objects. ${ }^{[14-16]}$

In previous works, most of the droplet rotations are driven by Lorentz forces, ${ }^{[17-19]}$ heat flow, ${ }^{[20-22]}$ surface structure, ${ }^{[23]}$ or the Marangoni force. ${ }^{[24]}$ With magnetically levitated and rotated

Dr. Z. Wang, Dr. X. Wang, Dr. Q. Miao, Prof. Y.-P. Zhao

State Key Laboratory of Nonlinear Mechanics

Institute of Mechanics

Chinese Academy of Sciences

15 Bei si huan xi Road, Beijing 100190, China

E-mail:yzhao@imech.ac.cn

Dr. Z. Wang, Dr. X. Wang, Dr. Q. Miao, Prof. Y.-P. Zhao

School of Engineering Science

University of Chinese Academy of Sciences

19 Yuquan Road, Beijing 100049, China

The ORCID identification number(s) for the author(s) of this article can be found under https://doi.org/10.1002/admi.202001756.

DOI: 10.1002/admi.202001756
However, the rotations of the objects in these works all lie on specific conditions or devices. Pimienta et al. ${ }^{[27,28]}$ found that water-saturated dichloromethane droplets can spontaneously spin on aqueous solutions of cetyltrimethylammonium bromide (CTAB). However, the lifetime of these drops is only a few tens of seconds. Despite these extensive works, realizing continuous, spontaneous, and stable droplet rotation without any external interference remains a challenge.

Spontaneous rotational droplets without any external forces are capable of taking energy from their environment and converting it into directed motion. In previous works, far-fromequilibrium systems exhibited a variety of temporal and spatial patterns, ${ }^{[25]}$ such as the spiral vortex of a bacterial suspension droplet. ${ }^{[29]}$ In these systems, the confining surfaces play crucial roles in the dynamics, transport, and order of internal active matter. ${ }^{[2-31]}$ Systems containing chemical reactions are often far from equilibrium, such as the displacement reaction. In these systems, do the internal products induce a droplet to move spontaneously under the action of a restricted surface?

In this article, we report a novel phenomenon in which an acid solution (AS) droplet spontaneously and stably rotates on the surface of an LM. This rotation is driven by the collective movement of bubbles generated in the droplet. It also demonstrates that non-living particulate matter can also selforganize and form an ordered spatial-temporal pattern under proper conditions. In the rotation process, the droplet exhibits a shape similar to that of the comma and rotates with a constant angular velocity that is determined by the experimental parameters, including the droplet radius, $\mathrm{pH}$, and composition of the LM. The formation of a comma shape is caused by the fluctuation of the droplet boundary, which is affected by the collective movements of bubbles that take up energy from the chemical 
reactions between the AS droplet and LM. The nonequilibrium Laplace pressure caused by the asymmetrical droplet shape also contributes to the rotation. The governing equation is deduced, and the main factors and characteristic angular velocity are obtained by dimensionless analysis. This research enables the directed conversion from chemical to mechanical energy and opens up a new way to make micro-motors with non-living materials.

To demonstrate this phenomenon, we examined the movements of an AS droplet (typical volume $V_{0} \approx 0.1 \mathrm{~mL}$ ) deposited on the surface of an LM at room temperature. The AS droplet is made of dilute sulfuric AS, and the $\mathrm{pH}$ is 1.68. The LM is composed of gallium (65\%) and indium (35\%). The $\mathrm{pH}$ value of the solution was measured using a $\mathrm{pH}$ meter (PHSJ-4, INESA Scientific Instrument Co. Ltd), and the average value of five measurements was taken. The LM was produced by Shenyang Northeast Nonferrous Metals Market Co. Ltd. In the experiments, a layer of oxide film is easily formed on the surface of the $\mathrm{LM}$ in air, which is an ideal 2D material. ${ }^{[1-2]}$ However, it is necessary to scrape it off to avoid the contact line pinning during the droplet movements and the hinder of chemical reaction between the droplet and the substrate. In the experiments, an LM is generally adopted instead of a solid metal, because on the surface of an LM, there is no obvious contact line pinning due to the liquid phase interface between the droplet and the substrate. This reduces the hindering of droplet movements. On the surface of a solid metal, such as a smooth zinc block, the chemical reactions result in a rough surface morphology, for example, sharp roughness or defects. These defects can severely impede droplet movement; ${ }^{[32]}$ a droplet on these surfaces generally stands still.

Photographs of a rotational droplet are shown in Figure 1a for two cycles. The duration is $\approx 1.4 \mathrm{~s}$, and the period is $\approx 0.58 \mathrm{~s}$. The angular velocity $\omega$ is $\approx 10.8 \mathrm{rad} \mathrm{s}^{-1}$, and the life time of this rotation is several minutes (see Video S1, Supporting Information). From the images, it can be seen that the droplets take on an asymmetric shape similar to a comma or yinyang, instead of a circular shape, in the process of rotation. The rotation caused by a comma shape is well known from the phenomenon that comma-shaped camphor fragments can spontaneously rotate in water. ${ }^{[33-35]}$ The asymmetric shape creates additional torque on the camphor fragments and promotes continuous rotation. ${ }^{[33-35]}$ In Figure 1a, the droplet rotation is analogous to the case of camphor fragments. The asymmetric shape causes the nonequilibrium Laplace pressure, which contributes to the droplet rotation. This is described in detail in Figure 4. When an AS droplet is deposited on the surface of the LM, the sulfuric acid in the droplets reacts with $\mathrm{Ga}$ in the LM. The equation for this chemical reaction is $2 \mathrm{Ga}+3 \mathrm{H}_{2} \mathrm{SO}_{4}=\mathrm{Ga}_{2}\left(\mathrm{SO}_{4}\right)_{3}+3 \mathrm{H}_{2} \uparrow$. These reactions produce numerous tiny bubbles. Under the action of buoyancy, these bubbles have a tendency to move outside the droplet. They take up chemical energy and convert it into directed motion. Therefore, the bubble in the droplet belongs to the active particles and has the characteristics of directional movement. ${ }^{[36]}$ This case is similar to the collective movement of Bacillus subtilis in suspension. Constrained by the physical boundary, the competition between radial confinements, selfpropulsion, steric interactions, and hydrodynamics robustly induces a steady single-vortex state. ${ }^{[29]}$ At the same time, the internal flow drives these bubbles to gather at the droplet boundary. The movement of agglomerated bubbles induces instability of the contact line and results in the symmetrybreaking of circular droplets and a comma shape formation, as shown in Figure 3. The tiny bubbles at the interface also decrease the hindering force of droplet rotation and cause significant slippage of the droplet. ${ }^{[37,38]}$ This is similar to the Leidenfrost effect, in which the droplet is levitated from the substrate by a vapor film. ${ }^{[39-41]}$ These factors together cause the droplet to rotate at an angular velocity greater than that of the bacterial suspension and related devices.

With respect to the rotation direction, the left- and right-handed rotations have the same probability of occurrence. Thus far, the occurrence of left or right rotation is stochastic. This is due to the fact that it is constructed by continuous chemical reactions and belongs to a dissipative structure. Images of the left- and righthanded rotations captured by a high-speed camera are shown in Figure $1 \mathrm{~b}$. The droplet volume was $0.5 \mathrm{~mL}$ and the $\mathrm{pH}$ was 1.48 . Under the same experimental conditions, the rotation periods are approximately the same. In Figure $1 \mathrm{~b}$, the left images present the left-handed rotation, and the period is $\approx 580 \mathrm{~ms}$ (also see Video S2, Supporting Information). In the right images, the right-handed rotation is shown, and the period is $\approx 560 \mathrm{~ms}$ (also see Video S3, Supporting Information). The rotation periods of the droplets in
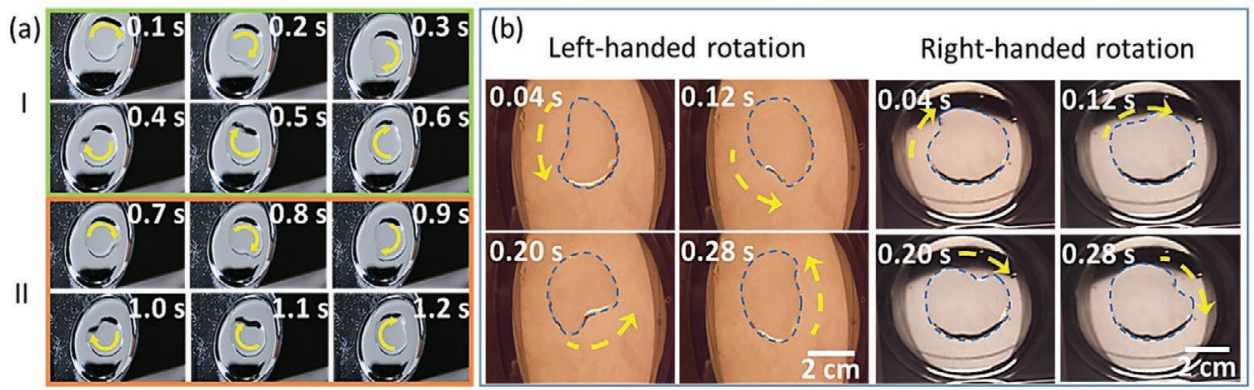

Figure 1. Rotation of an acid solution droplet on liquid metal. a) Photos of continuous rotation of a droplet. Two cycles of droplet rotation are shown. During the rotation, the droplet shows a yin-yang or comma shape and rotates in a fixed direction continuously. The yellow arrow points to the direction of rotation. This motion can last for several minutes. b) Left-handed and right-handed rotation of droplets taken with a high-speed camera. The droplet volume is $0.5 \mathrm{~mL}$, and the $\mathrm{pH}$ is 1.48 . The yellow arrows point to the rotation direction. The periods are $0.58 \mathrm{~s}$ (left-handed rotation) and $0.56 \mathrm{~s}$ (right-handed rotation), respectively. 
Figure 1a,b are approximately the same owing to the roughly equal experimental parameters.

The variation of the rotation angle $\varphi$, defined as the angle at which the droplet rotates clockwise with time, is shown in Figure 2a. A linear relation is exhibited, which indicates a constant angular velocity and stable rotation. The rotation period is $\approx 580 \mathrm{~ms}$ and the lifetime of this rotation is several minutes. In the experiments, the droplet on the LM exhibited an almost flat shape, which was determined by whether its radius was smaller or larger than the capillary length $k^{-1}$. For large droplets whose radius exceeds $k^{-1}$, the gravitational effect dominates. For the AS droplet on the LM, $k^{-1}$ is obtained as $k^{-1}=\left(\chi_{\mathrm{v}} / \rho \mathrm{g}\right)^{1 / 2}=2.749 \mathrm{~mm}$. The radius is $10^{-1} \mathrm{~m}$, which is larger than the capillary length. The thickness can be recast in terms of the capillary length $e=2 k^{-1} \sin \left(\theta_{\mathrm{e}} / 2\right)$. When $\theta_{\mathrm{e}}<1$, the thickness simplifies to $e=k^{-1} \theta_{\mathrm{e}} \cdot{ }^{[42]}$

The period of this rotation depends on the experimental parameters, including the droplet radius, $\mathrm{pH}$, and composition of the LM. In Figure $2 \mathrm{~b}-\mathrm{d}$, the effect of these parameters on the rotation period is shown. Figure $2 \mathrm{~b}$ shows the variation of $T$ with $\mathrm{pH}$. The rotation period changes from 0.3 to $0.6 \mathrm{~s}$, and increases linearly with $\mathrm{pH}$. Two radii of $R=6$ and $8 \mathrm{~mm}$ are used, and a larger droplet has a longer period. Figure $2 \mathrm{c}$ shows the effect of the droplet radius on the rotation period. The results indicate that $T$ increases linearly with $R$. This is because a large droplet has a relatively large rotational inertia, which leads to a small angular velocity if the driving force does not change significantly. Figure 2c shows the effect of the $\mathrm{Ga}$ content on $\mathrm{T}$. The results indicate that the content of $\mathrm{Ga}$ has a small effect on the rotation period. This is because the volume of the LM droplet is much larger than that of the AS droplet.
The Ga supply in the reactions is adequate for different components of the LM.

In Figure 3, the bubbles generated in the droplet and the process of comma shape formation are shown. In Figure 3a, a diagram is employed to illustrate the formation of a comma-shaped droplet. The entire process is roughly divided into three steps: initial circular droplet, shape deformation (symmetry-breaking), and comma shape formation. When an AS droplet is deposited on the surface of the LM, it initially maintains a circular shape, while chemical reactions occur at the interface. The bubbles generated by these reactions gather near the boundary owing to the internal flow. ${ }^{[43]}$ The interaction between bubbles causes the contact line to propagate outward due to the lack of inward bubble collisions, as shown in the second image. The forces $F_{\mathrm{bl}}, F_{\mathrm{br}}$, and $F_{\mathrm{bf}}$ represent the force exerted by adjacent bubbles on a bubble to the left, right, and front, respectively. With the force $F_{\mathrm{bf}}$, the droplet forms a forward part, which is shown in the third image. Subsequently, the outspread parts of the droplet expand outward again on both sides under the forces $F_{\mathrm{bf}}, F_{\mathrm{sl}}$ (to the left rotation), and $F_{\mathrm{br}}$ and $F_{\mathrm{sl}}$ (to the right rotation). $F_{\mathrm{sl}}$ represents the force exerted by the surface tension of the droplet to the left. This behavior results in a bifurcation of the droplet on the steering and the possibility of turning left or right. In this process, the symmetry of droplets is broken, and a new spatialtemporal pattern emerges under the action of bubble emergence and self-organization. The experimental demonstration of this process is shown in Figure 4. The continuous chemical reaction provides support for the stability of the new spatialtemporal pattern of the droplet. The conversion from chemical to mechanical energy is at the origin of a wide variety of
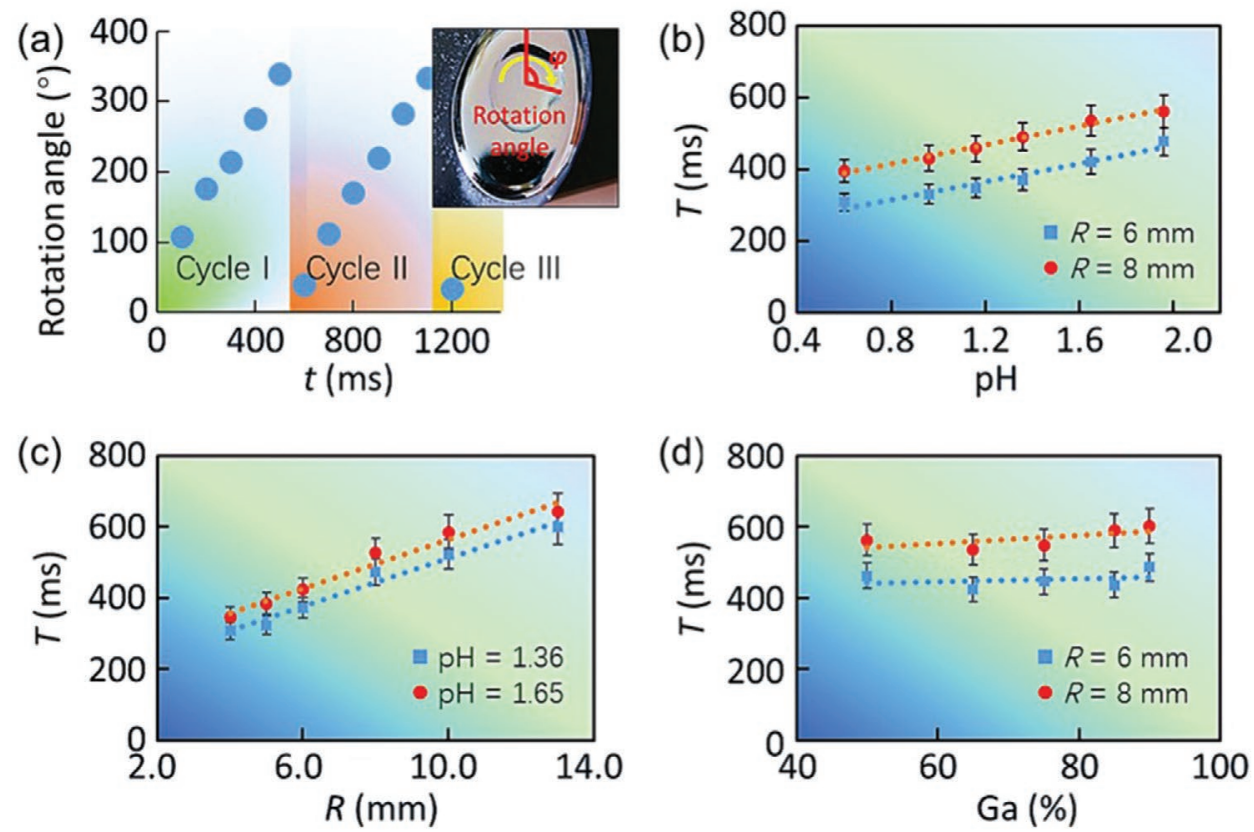

Figure 2. The periods of droplet rotation. a) Variation of rotation angle with time (droplet radius: $1 \mathrm{~cm}$; pH: 1.48 ; $\mathrm{Ga} 65 \%$; and In $35 \%$ ). The results show an approximately linear relationship between rotation angle and time. This indicates a constant angular velocity during the rotation. The period is $\approx 0.58 \mathrm{~s}$. The angular velocity $(\omega)$ is $10.8 \mathrm{rad} \mathrm{s}^{-1}$. b) The effect of $\mathrm{pH}$ on the period of droplet rotation. As the $\mathrm{pH}$ increases, the period increases. The droplet of $6 \mathrm{~mm}$ has a smaller period than that of $8 \mathrm{~mm}$. c) The rotation period increases with droplet radius. The Ga content is $65 \%$. d) The effect of Ga content. It has little effect on the rotation period. 
(a)
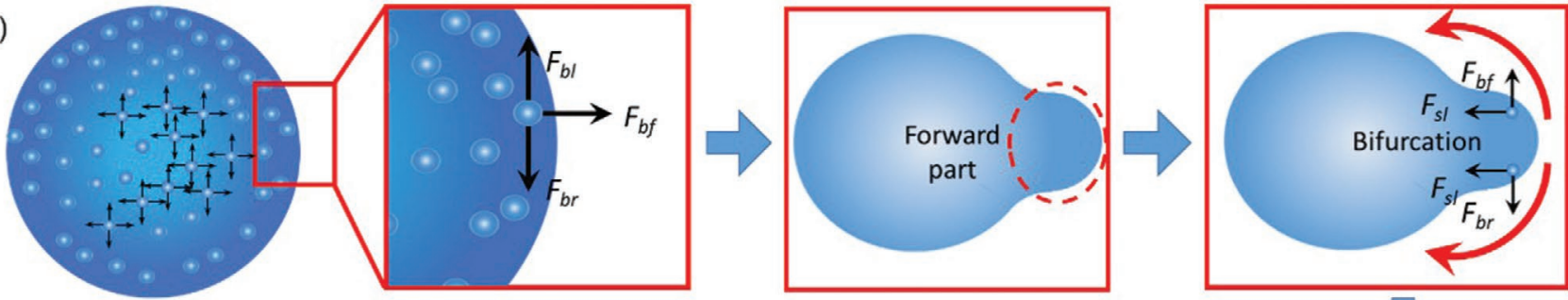

(b) Bubbles
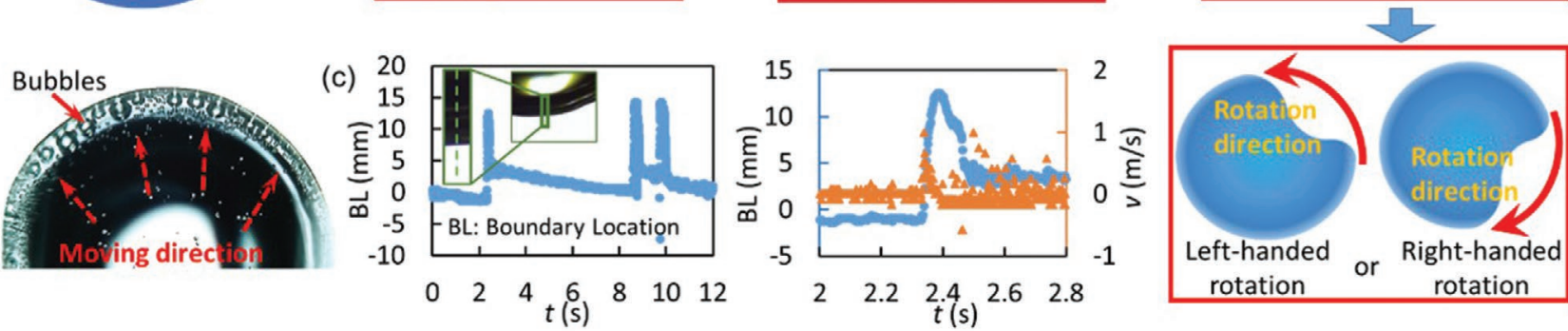

Figure 3. Emergence of a new spatial-temporal pattern driven by collective motion of bubbles. a) Diagram of the formation of a comma shape along with the self-organization of bubbles and symmetry-breaking of the round droplet. In this process, the nonequilibrium of the interaction force between bubbles results in the droplet boundary propagating outward and forming a comma shape. The direction in which the droplet rotates is a bifurcation. This leads to the randomness of droplet rotation. b) The bubbles generated in the droplet. Red arrows point to the motion direction of bubbles. Under the internal flow fields, these bubbles tend to gather at the boundary of the droplet. c) The fluctuation of droplet boundary location affected by the bubble motion. For the convenience of observation, the droplet $(\mathrm{pH} \approx 2.26)$ and $\mathrm{LM}(65 \% \mathrm{Ga}, 35 \% \mathrm{In})$ are adopted because the boundary motions are relatively mild under this condition. For the illustration of droplet boundary motion, a random point on the contact line is selected, as shown in the insert image. The other positions have similar motion rules. At the droplet boundary, the contact line moves outward rapidly. Then, the contact line is quickly pulled back and forms a pulse motion. As the reaction intensity increases, the effect of random fluctuation on the spatial-temporal patterns becomes significant. This phenomenon verifies the existence of the forward part.

dissipative structures, such as regular convective cells and interfacial turbulences. ${ }^{[33,44]}$

In Figure $3 \mathrm{~b}$, the bubbles generated in the droplet are shown. The red arrows indicate the moving direction of the bubbles. This phenomenon indirectly shows the flow field from the center to the boundary inside the droplet under chemical reaction. In Figure 3c, the fluctuation of the droplet boundary is shown. The boundary location was recorded, and
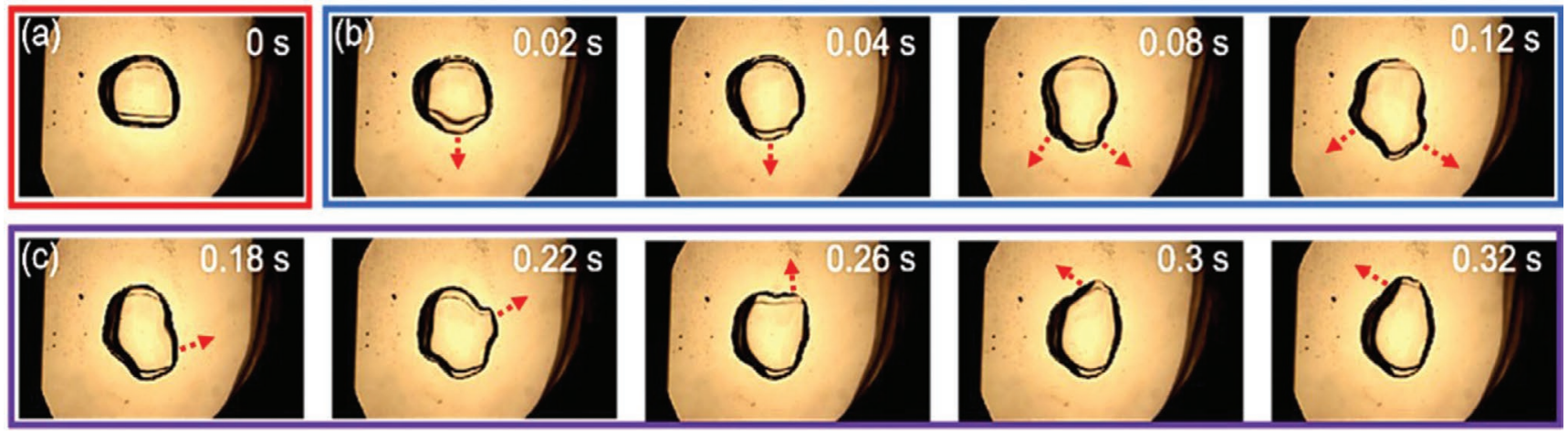

$0.3 \mathrm{~s}$

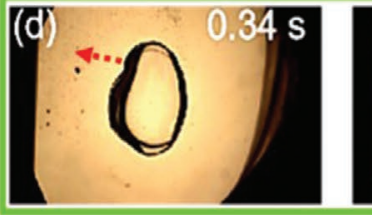

Initial stage
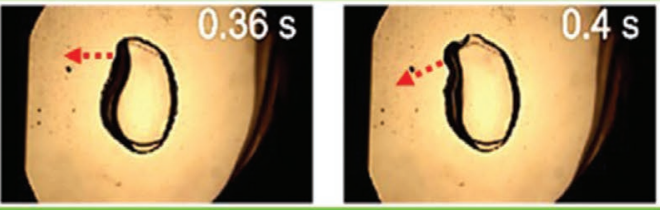

Deformation stage

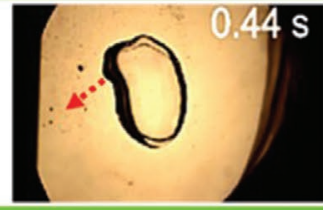

Direction finding stage

\section{$\square$ Direction determination stage}

Figure 4. The process of comma shape formation. a) The initial stage. The initial shape of a droplet after it drops on the surface of LM. b) The deformation stage. Parts of contact line or the whole contact line extends outside rapidly due to the chemical reaction. This behavior results in an irregular shape of droplet. c) The direction finding stage. The two sides of expanding liquid extend to the left and right, respectively. This movement leads to different directions of rotation. d) The direction determination stage. Once one side of the expanding liquid is stronger than another, the direction is determined. 
the velocity was measured. One point at the contact line was selected to illustrate this motion. The point is selected randomly. The movements of the contact line in other positions have similar moving behaviors. For the convenience of observation, a droplet $(\mathrm{pH} \approx 2.26)$ and LM $(65 \% \mathrm{Ga}, 35 \% \mathrm{In})$ were adopted. The boundary motions are relatively mild owing to the weak chemical reactions. From the results, it can be seen that the contact line moves outward rapidly and is then pulled back quickly. It moves as a pulse. Its velocity is on the order of $10^{0} \mathrm{~m} \mathrm{~s}^{-1}$. A pulse is completed in $\approx 0.2 \mathrm{~s}$. The inserted images show the selected position of the droplet boundary motion. In Figure 3c, the contact line completed three pulses in $12 \mathrm{~s}$ owing to the weak chemical reaction. As the reaction intensity increases, the fluctuations may become more frequent and violent. This behavior provides the essential conditions for droplet motion from disorder to order and determines the final spatial-temporal patterns of the droplet. The rapid outward movement verifies the existence of the forward part.

In Figure 4, the experimental demonstration of the proposed model in Figure 3a is shown. In this process, the comma shape formation is divided into four stages, including the initial stage, deformation stage, direction finding stage and direction determination stage. In the first stage, the droplet is stationary at the moment that it falls on the surface of LM (Figure 4a). After that, the droplet boundary begins to expand outward and the symmetry of droplet is broken, as shown in Figure 4b. Here, one more typical case is taken, that is, parts of boundary extend outward for facilitating analysis. In most cases, droplet expansion occurs everywhere along the contact line. Figure $4 \mathrm{c}$ depicts the direction finding stage. At this stage, the outspread parts of the droplet expand outward again on both sides. This behavior results in the possibility of left-handed or right-handed rotation of droplet. The rotation direction is not determined. In Figure $4 \mathrm{~d}$, the two protruding parts continue to move outward. Once the movement of one side is stronger than another, the droplet forms a comma shape that is tilted to the stronger side (direction determination stage).
After the formation of the comma shape of the droplet, the Laplace pressure becomes important, as the contact line is asymmetric. The droplet rotates continuously under the action of the Laplace pressure (Figure 5). This is similar to the phenomenon in which a camphor fragment designed as a comma can spontaneously rotate on water. In this case, the convex and concave parts of the camphor fragment, that is, the positive curvature and negative curvature part, produce torque on the camphor fragment and cause it to rotate. ${ }^{[3-35]}$ In Figure $5 \mathrm{a}$, the curvature of the comma-shaped droplet varies at different points along the contact line. The different curvatures lead to unequal Laplace pressures, which are generally given by equation $\Delta p=\gamma\left(1 / r_{1}+1 / r_{2}\right)$, where $\gamma$ is the surface tension of the liquid, and $r_{1}$ and $r_{2}$ are two orthogonal curvatures of the curved liquid surface, respectively. In Figure $5 \mathrm{a}$, the difference in Laplace pressure is mainly located in places where the curvature changes significantly, that is, Regions I and II. In the two sections, the droplet has a positive curvature and a negative curvature, respectively. In the enlarged images of Regions I and II, it can be seen that the Laplace pressure points to the inside of the droplet in Region I and to the outside of the droplet in Region II. This causes the droplet to produce inward and outward forces, $F_{1}$ and $F_{2}$, respectively. In Figure $5 \mathrm{a}$, both forces exert a torque $\left(T_{1}\right.$ and $\left.T_{2}\right)$ that induces the right-handed rotation of the droplet. This is analogous to the rotation of the commashaped camphor fragments under the action of an unbalanced Laplace pressure. ${ }^{[3-35]}$ In Figure $5 b$, the case is similar. The torques, caused by the inward and outward forces, $F_{1}$ and $F_{2}$, result in a continuously left-handed rotation.

In order to obtain the governing equation of droplet rotation and the main factors affecting the rotation period, the NavierStokes equations are employed. Here, the viscous force, inertial force, and surface tension need to be considered. The droplet motion is a rotational and incompressible flow, namely, $\nabla \cdot \boldsymbol{\omega}=0$ and $\nabla \cdot \boldsymbol{v}=0$, where $\boldsymbol{\omega}$ and $\boldsymbol{v}$ are the angular velocity and velocity, respectively. Generally, the Navier-Stokes equations are expressed as follows (a)

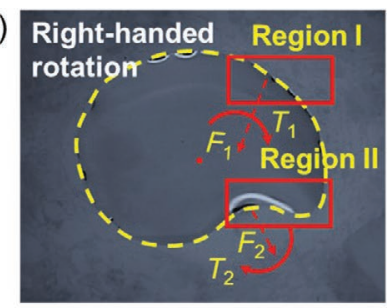

(b)

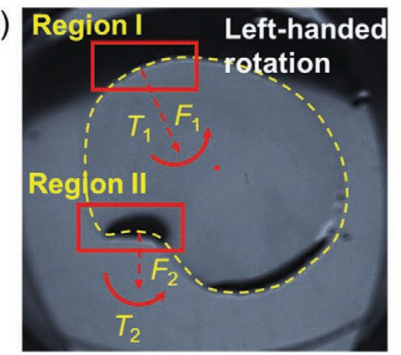

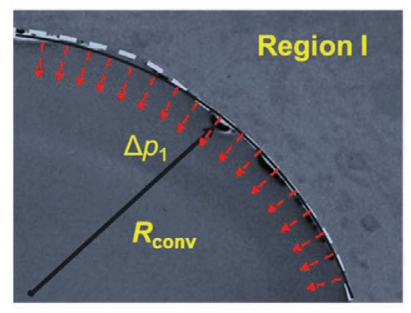

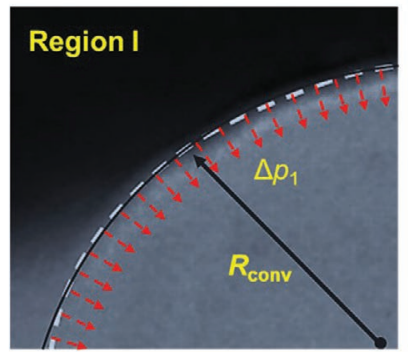

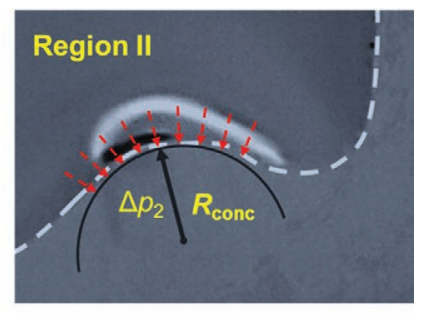

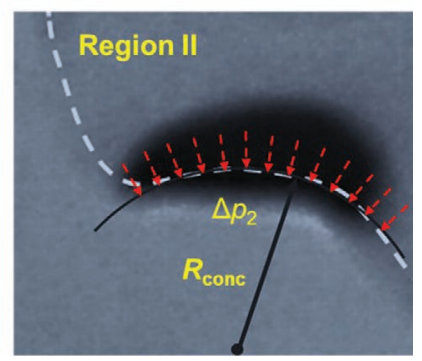

Figure 5. The Laplace pressure drives the comma-shaped droplet to continuously rotate. a) Diagram of comma-shaped AS droplet of right-handed rotation. The droplet has a positive curvature in Region I and a negative curvature in Region II. The Laplace pressure generated in Region I and II collectively exert a torque on the droplet, causing it to rotate continuously. b) Diagram of comma-shaped droplet of left-handed rotation. 
$\rho \frac{\mathrm{d} \boldsymbol{v}}{\mathrm{d} t}=-\nabla p+\mu \Delta \mathbf{v}+\rho \boldsymbol{g}$

where $\rho, g, p$, and $\mu$ are the density of the fluid, acceleration of gravity, surface pressure, and viscosity of the fluid, respectively. In this rotation, the surface force term only consists of the surface tension and can be expressed as $p=-\chi\left(1 / r_{1}+1 / r_{2}\right)$. By taking the curl of the Navier-Stokes equations and ignoring the compressibility, the governing equation can be obtained as

$\frac{\mathrm{d} \omega}{\mathrm{d} t}=\frac{\gamma}{\rho} \nabla \times(\nabla \alpha)+\nabla \times(v \Delta \mathbf{v})+(\omega \cdot \nabla) \boldsymbol{v}$

where $\alpha=1 / r_{1}+1 / r_{2}$, and $v$ is the kinematic viscosity of the fluids. For an acid droplet on the surface of LM, many bubbles are produced in the interface. From previous works, ${ }^{[37,39]}$ nanoor micro-bubbles in the interface can lead to an apparent slip of the liquid. Therefore, when dealing with this problem, we can regard it as a slip boundary condition.

From the governing equation, the relevant dimensionless numbers and characteristic angular velocity can also be obtained. First, we need to nondimensionalize the governing equation. The factors that need to be dimensionless include rotation angular velocity, time, velocity, length, the Hamiltonian operator, and the Laplace operator, namely, $\omega=\omega^{*} \Omega, t=t^{*} T$, $v=v^{*}(L / T), \alpha=\alpha^{*} / L, \nabla=\nabla^{*} / L$, and $\Delta=\Delta^{*} / L^{2}$. Taking these dimensionless factors into the governing equation, we obtain

$\underset{(1)}{\frac{\Omega}{T}} \frac{\mathrm{d} \omega^{*}}{\mathrm{~d} t^{*}}=\underbrace{\frac{\gamma}{\rho L^{3}}}_{(2)} \nabla^{*} \times\left(\nabla^{*} \alpha^{*}\right)+\underbrace{\frac{v}{L^{2} T}}_{(3)} \nabla^{*} \times\left(\Delta^{*} \boldsymbol{v}^{*}\right)+\underbrace{\frac{\Omega}{T}}_{(1)}\left(\omega^{*} \cdot \nabla^{*}\right) \mathbf{v}^{*}$

where (1), (2), and (3) represent the dimensional parts extracted from each term. By comparing (1)-(2), (1)-(3), and (2)-(3), we can obtain the dimensionless numbers of rotational liquids, including the Weber number, $W e=\rho \Omega^{2} L^{3} / \gamma$, the Reynolds number, $R e=\Omega L^{2} / \gamma$, and the capillary number, $C a=\mu L / \gamma T$. The capillary number is consistent with that of the irrotational liquids, while the angular velocity replaces the velocity $v$ in the Weber number and the Reynolds number. For the AS droplets on $\mathrm{LM}$, the radius scales as $10^{-2} \mathrm{~m}$. Thus, the values of these dimensionless numbers are $\mathrm{We} \approx 1.4 \times 10^{0}, \mathrm{Re} \approx 10^{3}$, and $C a \approx 2.7 \times 10^{-3}$. To compare the effect of each factor on the droplet rotation, the dimensionless equation is written in the form of the Weber number as

$\underbrace{W e}_{10^{0}} \frac{\mathrm{d} \omega^{*}}{\mathrm{~d} t^{*}}=\underbrace{1}_{10^{0}} \nabla^{*} \times\left(\nabla^{*} \alpha^{*}\right)+\underbrace{\operatorname{Ca}}_{10^{-3}} \nabla^{*} \times\left(\Delta^{*} \mathbf{v}^{*}\right)+\underbrace{W e}_{0^{0}}\left(\omega^{*} \cdot \nabla^{*}\right) \mathbf{v}^{*}$

The capillary term in Equation (4) can be ignored because it is much smaller than the other terms. Therefore, the rotation of the droplet is mainly controlled by the internal force and surface tension. The characteristic angular velocity can be obtained as $\Omega=\left(\gamma / \rho L^{3}\right)^{1 / 2}$. For the AS droplets used in the experiments, the parameters are $\gamma=72 \mathrm{mN} \mathrm{m}^{-1}, \mu=0.89 \mathrm{mPa}$ s, and $L=2 \mathrm{~cm}$. The angular velocity is obtained as $\approx 10^{1} \mathrm{rad} \mathrm{s}^{-1}$, which is the same order of magnitude as the experimental results. These results indicate the effectiveness of the governing equation and dimensional analysis. It is also clarified that the movement of the droplet is mainly controlled by inertial force and surface tension.

In summary, the spontaneous rotation of an AS droplet on the surface of an LM under the effect of bubble collective movement was discovered. This phenomenon indicates that non-living particles can spontaneously form ordered motion under certain conditions, not only limited to living particles and artificial particles. From the experimental results, it can be seen that the droplets rotate with a faster angular velocity under the action of bubble collective movements, compared to the motion of living particles. The entire process of this rotation includes the comma-shape formation under the effect of bubble movement and continuous rotation driven by the Laplace pressure. The comma shape formation originates from the fluctuation of the droplet boundary affected by the bubble movements. From disorder to order, the emergence and selforganization of bubbles and the symmetry-breaking of droplets occur. The periods of droplet rotation affected by experimental parameters, including droplet radius, $\mathrm{pH}$, and composition of the LM, were measured. The governing equations employing the Navier-Stokes equations are deduced and discussed. The dominant factors and characteristic angular velocity of the droplet rotation are obtained with dimensionless analysis. The results agree with the experimental data.

\section{Supporting Information}

Supporting Information is available from the Wiley Online Library or from the author.

\section{Acknowledgements}

This work was supported by the National Natural Science Foundation of China (NSFC) for "Multiscale Problems in Nonlinear Mechanics" (Grant 11988102), as well as NSFC Grants 12032019, 51861145314, and 11872363; the Chinese Academy of Sciences (CAS) Key Research Program of Frontier Sciences (Grant QYZDJ-SSW-JSC019), and the CAS Strategic Priority Research Program (Grant XDB22040401).

\section{Conflict of Interest}

The authors declare no conflict of interest.

\section{Keywords}

bubbles, collective motion, liquid metals, self-propulsion, self-rotating droplets, spontaneous rotation

Received: October 13, 2020

Revised: November 11, 2020

Published online: December 13, 2020

[1] T. Daeneke, K. Khoshmanesh, N. Mahmood, I. A. de Castro, D. Esrafilzadeh, S. J. Barrow, M. D. Dickey, K. Kalantar-zadeh, Chem. Soc. Rev. 2018, 47, 4073. 
[2] G. Bo, L. Ren, X. Xu, Y. Du, S. Dou, Adv. Phys.: X 2018, 3, 1446359.

[3] J. Liu, L. Sheng, Z. Z. He, Liquid Metal Soft Machines: Principles and Applications, Springer, New York 2018.

[4] H. Chang, R. Guo, Z. Sun, H. Wang, Y. Hou, Q. Wang, W. Rao, J. Liu, Adv. Mater. Interfaces 2018, 5, 1800571.

[5] L. Hu, L. Wang, Y. Ding, S. Zhan, J. Liu, Adv. Mater. 2016, 28, 9210.

[6] Y. Ding, Doctoral Thesis, Technical Institute of Physics and Chemistry, Chinese Academy of Sciences, Beijing, China 2019.

[7] K. C. Park, P. Kim, A. Grinthal, N. He, D. Fox, J. C. Weaver, J. Aizenberg, Nature 2016, 531, 78.

[8] N. J. Cira, A. Benusiglio, M. Prakash, Nature 2015, 519, 446.

[9] O. D. Velev, B. G. Prevo, K. H. Bhatt, Nature 2003, 426, 515.

[10] M. J. Kreder, J. Alvarenga, P. Kim, J. Aizenberg, Nat. Rev. Mater. 2016, 1, 15003.

[11] M. S. Feiz, R. M. Namin, A. Amjadi, Phys. Rev. E 2015, 92, 033002.

[12] J. Wu, S. Y. Tang, T. Fang, W. Li, X. Li, S. Zhang, Adv. Mater. 2018, 30, 1805039.

[13] J. A. F. Plateau, Annual Report of the Board of Regents of the Smithsonian Institution 1863, p. 270

[14] P. Lacerda, D. C. Jewitt, Astron. J. 2007, 133, 1393.

[15] H. Saarikoski, S. M. Reimann, A. Harju, M. Manninen, Rev. Mod. Phys. 2010, 82, 2785.

[16] D. Foresti, D. Poulikakos, Phys. Rev. Lett. 2014, 112, 024301.

[17] S. A. Lira, J. A. Miranda, R. M. Oliveira, Phys. Rev. E 2010, 82, 036318.

[18] L. Liao, R. J. A. Hill, Phys. Rev. Lett. 2017, 119, 114501.

[19] R. J. A. Hill, L. Eaves, Phys. Rev. E 2010, 81, 056312.

[20] A. Bouillant, T. Mouterde, P. Bourrianne, A. Lagarde, C. Clanet, D. Quéré, Nat. Phys. 2018, 14, 1188.

[21] J. Ignés-Mullol, G. Poy, P. Oswald, Phys. Rev. Lett. 2016, 117, 057801.

[22] P. Oswald, A. Dequidt, Phys. Rev. Lett. 2008, 100, 217802.

[23] H. Li, W. Fang, Y. Li, Q. Yang, M. Li, Q. Li, X. Q. Feng, Y. Song, Nat. Commun. 2019, 10, 950.

[24] A. S. Basu, Y. B. Gianchandani, J. Micromech. Microeng. 2008, 18, 115031.
[25] Y. Koyano, M. Gryciuk, P. Skrobanska, M. Malecki, Y. Sumino, H. Kitahata, J. Gorecki, Phys. Rev. E 2017, 96, 012609.

[26] O. Lehmann, Ann. Phys. 1900, 2, 649.

[27] V. Pimienta, C. Antoine, Curr. Opin. Colloid Interface Sci. 2014, 19, 290.

[28] V. Pimienta, M. Brost, N. Kovalchuk, S. Bresch, O. Steinbock, Angew. Chem., Int. Ed. 2011, 50, 10728.

[29] H. Wioland, F. G. Woodhouse, J. Dunkel, J. O. Kessler, R. E. Goldstein, Phys. Rev. Lett. 2013, 110, 268102.

[30] K. Kruse, J. F. Joanny, F. Jülicher, J. Prost, K. Sekimoto, Phys. Rev. Lett. 2004, 92, 078101.

[31] A. Sokolov, I. S. Aranson, J. O. Kessler, R. E. Goldstein, Phys. Rev. Lett. 2007, 98, 158102.

[32] Z. Wang, K. Lin, Y. P. Zhao, J. Colloid Interface Sci. 2019, 552, 563.

[33] Y. Koyano, M. Gryciuk, P. Skrobanska, M. Malecki, Y. Sumino, H. Kitahata, J. Gorecki, Phys. Rev. E 2017, 96, 012609.

[34] Y. Koyano, N. J. Suematsu, H. Kitahata, Phys. Rev. E 2019, 99, 022211.

[35] S. Nakata, Y. Iguchi, S. Ose, M. Kuboyama, T. Ishii, K. Yoshikawa, Langmuir 1997, 13, 4454.

[36] C. Bechinger, R. D. Leonardo, H. Löwen, C. Reichhardt, G. Volpe, G. Volpe, Rev. Mod. Phys. 2016, 88, 045006

[37] P. G. de Gennes, Langmuir 2002, 18, 3413.

[38] S. Granick, Y. Zhu, H. Lee, Nat. Mater. 2003, 2, 221.

[39] D. Quéré, Annu. Rev. Fluid Mech. 2013, 45, 197.

[40] Z. H. Wu, W. H. Chang, C. Sun, Int. J. Therm. Sci. 2018, 129, 254.

[41] Y. P. Zhao, Physical Mechanics of Surfaces and Interfaces, Science Press, Beijing 2012.

[42] R. Tadmor, R. Das, S. Gulec, J. Liu, H. E. N'guessan, M. Shah, P. S. Wasnik, S. B. Yadav, Langmuir 2017, 33, 3594.

[43] I. Tuval, L. Cisneros, C. Dombrowski, C. W. Wolgemuth, J. O. Kessler, R. E. Goldstein, Proc. Natl. Acad. Sci. USA 2005, 102, 2277.

[44] V. Pimienta, C. Antoine, Curr. Opin. Colloid Interface Sci. 2014, 19, 290. 